\title{
The Difference of Lower Urinary Tract Symptoms Between Sympathetic Hyperactive and Hypoactive Men
}

\author{
Dong Geun Oh, Dae Sung Cho ${ }^{1}$, In Suk Yun², Kuk Bin Lee ${ }^{2}$, Jong Bo Choi², Jung Hwan Lee \\ Son \& Oh Urologic Clinic, Suwon; \\ ${ }^{1}$ Department of Urology, Bundang Jesaeng Hospital, Seongnam; \\ ${ }^{2}$ Department of Urology, Ajou University School of Medicine, Suwon, Korea
}

\begin{abstract}
Purpose: Heart rate variability (HRV) is a tool used to measure autonomic nervous function; however, there is no evidence that it can be used to define sympathetic hyperactivity in men with lower urinary tract symptoms (LUTS). We suspected that LUTS would differ between sympathetic hyperactive and hypoactive patients. Therefore, we measured HRV and divided the LUTS patients into two groups, a sympathetic hyperactive group and a sympathetic hypoactive group according to the low frequency/high frequency $(\mathrm{LF} / \mathrm{HF})$ ratio and made clinical comparisons between the groups.

Methods: A total of 43 patients with symptomatic LUTS (International Prostate Symptom Score [IPSS] over 8) and 49 healthy volunteers were enrolled. No subjects had diseases that could affect the autonomic nervous system, such as diabetes or hypertension. Electrocardiographic signals were obtained from subjects in the resting state and HRV indexes were calculated with spectral analyses. We divided the LUTS patients into two groups by an LF/HF ratio of 1.9, which was the median value in the healthy volunteers, and compared the differences in clinical characteristics, IPSS, prostate-specific antigen (PSA), and transrectal ultrasound (TRUS) results. The parameters were compared by independent sample t-test by use of SPSS ver. 19.

Results: There were no significant differences in age, serum PSA, or volume of the prostate between the 2 LUTS groups. However, analyzing IPSS questionnaires between two groups showed that there were significant differences in mean of Q2 score (frequency) and storage symptom score $([\mathrm{Q} 2+\mathrm{Q} 4+\mathrm{Q} 7] / 3)(\mathrm{P}<0.05)$.

Conclusions: We suggest that an imbalance of autonomic nervous system activity may be a factor that evokes varieties of symptoms in men with LUTS. LUTS patients with hypoactive sympathetic tone may suffer from frequency and storage symptoms.
\end{abstract}

Keywords: Prostatic hyperplasia; Autonomic nervous system; Lower urinary tract symptoms; Heart rate variability

\section{INTRODUCTION}

Lower urinary tract symptoms (LUTS) are relatively common in aging men, and they have been known to greatly affect quality of life [1]. Because the number of men with benign prostatic hyperplasia (BPH) and LUTS continues to increase, it is important that the underlying pathogenesis of the disease is well understood. The relationship between LUTS and objective measures of $\mathrm{BPH}$, such as prostate size and urodynamic parameters, has proved difficult to determine [2].

Heart rate variability (HRV) is widely used as a noninvasive tool that shows the balance of the cardiovascular system controlled by the sympathetic and parasympathetic divisions of the autonomic nervous system (ANS) [3]. HRV is related to many urologic diseases, such as erectile dysfunction, female incontinence and chronic prostatitis [2,4-6]. Recent studies have shown a correlation between ANS tone and LUTS, and with metabolic syndrome, which can affect ANS tone and also shows a correlation with BPH $[7,8]$. However, not all patients with LUTS have sympathetic hyperactivity. Therefore, we investigated the clinical differences between LUTS patients with sympathetic hyperactivity and sympathetic hypoactivity.
Corresponding author: Jung Hwan Lee

Department of Urology, Ajou University School of Medicine, 206 Worldcup-ro, Yeongtong-gu, Suwon 443-721, Korea

Tel: +82-31-219-7332 / Fax: +82-31-219-5276 / E-mail: urolee79@gmail.com Submitted: February 27, 2013 / Accepted after revision: March 30, 2013
This is an Open Access article distributed under the terms of the Creative Commons Attribution Non-Commercial License (http://creativecommons.org/licenses/by-nc/3.0/) which permits unrestricted non-commercial use, distribution, and reproduction in any medium, provided the original work is properly cited. 


\section{MATERIALS AND METHODS}

We selected 43 patients who visited an outpatient clinic for LUTS and 49 healthy volunteers who visited the family medicine outpatient clinic for a general health examination. No subjects had diseases that could affect the ANS, such as diabetes, hypertension, coronary heart disease, or any malignancy. No subjects had a history of surgery, irradiation, or pelvic trauma. All subjects answered an International Prostate Symptom Score (IPSS) questionnaire and patients with an IPSS $\geq 8$ were enrolled. We divided the LUTS patients into two groups (groups 1 and 2) by a low frequency/high frequency (LF/HF) ratio of 1.9, which was the median value in the healthy volunteers, and compared the clinical characteristics, IPSS, prostate-specific antigen (PSA), and transrectal ultrasound (TRUS) results between the two groups.

\section{HRV Measurement}

HRV was measured by using 2 methods: time domain and frequency domain analysis. The parameters of the time domain analysis were the mean heart rate in bpm, the standard deviation of the N-N interval (SDNN), and the square root of the mean squared difference of successive N-N intervals (RMSSD). SDNN reflects all of the cyclic components responsible for variability in the recording period and RMSSD represents parasympathetic activity. The parameters of the frequency domain analysis were total power (TP), very low frequency (VLF), LF, $\mathrm{HF}$ and LF/HF ratio. The HF peak of the spectrum $(0.15$ to 0.40 $\mathrm{Hz}$ ) represents parasympathetic activity, whereas the LF peak (0.04 to $0.15 \mathrm{~Hz}$ ) represents sympathetic activity. Thus, the LF/ HF ratio represents the ANS balance (sympathetic to parasympathetic).

We used a system developed in-house for HRV acquisition and signal processing to measure ANS activity. We confirmed that bladders were filled with more than $100 \mathrm{~mL}$ of urine before testing by ultrasonography to represent the physiological state. All subjects were restricted in their consumption of tea and coffee, cigarette smoking, and medication use (e.g., beta-receptor agonists or antagonists). After 30 minutes of rest, each patient underwent electrocardiographic signal recording (SA-3000P, Medicore Co., Seoul, Korea) for 5 minutes while sitting. With the patients breathing normally, we calculated the mean heart rate, SDNN and RMSSD. We also determined the resting sympathetic and parasympathetic heartbeat rate modulation by using frequency domain methods, including TP, VLF, LF, HF, and
LF/ HF ratio. HRV parameters were compared by using the independent sample t-test.

\section{Statistical Analysis}

Statistics were performed by using the IBM SPSS ver. 19.0 (IBM Co., Armonk, NY, USA). The data were presented as means \pm standard deviations. Comparisons between the two groups were performed with the independent t-test. P-values less than 0.05 were considered to be statistically significant.

\section{RESULTS}

The statistical analysis showed no significant differences between the control (healthy volunteers) and LUTS group in age, body mass index, serum PSA, or volume of the prostate (Table 1). Sympathetic hypoactive LUTS patients who LF/HF ratio below 1.9 were group 1 , and sympathetic hyperactive LUTS patients who LF/HF ration over 1.9 were group 2. The results of the analysis of the parameters of the IPSS questionnaire in LUTS patients (groups 1 and 2) are shown in Table 2. There were significant differences in mean of IPSS Q2 score (frequent urination) and storage symptom score ([Q2+Q4+Q7]/3) of IPSS according to ANS balance $(\mathrm{P}<0.05)$. Sympathetic hypoactive patients suffer from more severe storage symptoms especially frequency than sympathetic hyperactive patients.

\section{DISCUSSION}

The extended underlying pathophysiology leading to LUTS remains unclear, although it is increasingly recognized that the causes of LUTS extend well beyond prostate enlargement and

Table 1. Clinical characteristics between control and LUTS group

\begin{tabular}{lccc}
\hline & $\begin{array}{c}\text { Control group } \\
(\mathrm{n}=49)\end{array}$ & $\begin{array}{c}\text { LUTS group } \\
(\mathrm{n}=43)\end{array}$ & P-value \\
\hline Age $(\mathrm{yr})$ & $61.4 \pm 10.4$ & $58 \pm 7.8$ & 0.40 \\
Body mass index $\left(\mathrm{kg} / \mathrm{m}^{2}\right)$ & $24.5 \pm 3.4$ & $24.3 \pm 3.0$ & 0.87 \\
Total IPSS & $23 \pm 8.1$ & $17.3 \pm 6.9$ & 0.09 \\
PSA (ng/mL) & $2.67 \pm 2.0$ & $1.45 \pm 1.5$ & 0.15 \\
TRUS $(\mathrm{mL})$ & $31.41 \pm 9.1$ & $32.7 \pm 13.8$ & 0.82 \\
\hline
\end{tabular}

Values are presented as mean \pm standard deviation.

LUTS, lower urinary tract symptoms; IPSS, International Prostate Symptom Score; PSA, prostate-specific antigen; TRUS, transrectal ultrasonography. 
Table 2. The results of characteristics and IPSS parameters in two groups of LUTS patients by sympathetic activity

\begin{tabular}{lccc}
\hline Variable & $\begin{array}{c}\text { Group 1 } \\
(\mathrm{n}=24)\end{array}$ & $\begin{array}{c}\text { Group 2 } \\
(\mathrm{n}=19)\end{array}$ & P-value \\
\hline Age $(\mathrm{yr})$ & $59.6 \pm 9.3$ & $58.8 \pm 7.8$ & 0.25 \\
PSA (ng/mL) & $1.77 \pm 1.68$ & $1.46 \pm 1.73$ & 0.67 \\
TRUS (mL) & $29.4 \pm 9.1$ & $32.2 \pm 12.0$ & 0.21 \\
IPSS parameters & & & \\
Q1 & $3.46 \pm 1.77$ & $2.58 \pm 1.84$ & 0.27 \\
Q2 & $3.67 \pm 1.63$ & $2.79 \pm 1.62$ & $\left.0.04^{\mathrm{a}}\right)$ \\
Q3 & $3.08 \pm 1.86$ & $2.95 \pm 1.81$ & 0.87 \\
Q4 & $2.83 \pm 1.99$ & $2.32 \pm 1.89$ & 0.74 \\
Q5 & $4.08 \pm 1.28$ & $3.79 \pm 1.32$ & 0.54 \\
Q6 & $3.13 \pm 1.85$ & $2.53 \pm 1.35$ & 0.41 \\
Q7 & $2.83 \pm 1.49$ & $2.34 \pm 1.46$ & 0.35 \\
Total IPSS score & $23.08 \pm 7.48$ & $19.26 \pm 7.37$ & 0.08 \\
Quality of life & $4.58 \pm 0.97$ & $4.16 \pm 0.90$ & 0.15 \\
Voiding Sx score & & & \\
$\quad$ (Q1+Q3+Q5+Q6)/4 & $3.29 \pm 1.35$ & $2.90 \pm 1.36$ & 0.39 \\
Storage Sx score & & & \\
(Q2+Q4+Q7)/3 & $3.02 \pm 1.44$ & $2.11 \pm 1.26$ & $0.03^{\mathrm{a})}$ \\
\hline
\end{tabular}

Values are presented as mean \pm standard deviation.

Group 1, patients with LF/HF below 1.9; Group 2, patients with LF/HF 1.9 and over 1.9; IPSS, International Prostate Symptom Score; PSA, prostate-specific antigen; TRUS, transrectal ultrasonography; Sx, symptom; $\mathrm{Q}$, question number.

${ }^{\text {a) }} \mathrm{P}<0.05$.

bladder outlet obstruction [9]. The multiple pathways leading to the onset and progression of LUTS not only complicate diagnosis, but also limit the overall effectiveness and satisfaction of targeted symptom management strategies [10]. The lower urinary tract is generally under the control of the ANS, is innervated by 3 sets of peripheral nerves (parasympathetic, sympathetic, and somatic nervous systems), and contains afferent and efferent motor axons [11].

HRV depends on the influence of sympathetic and vagal activity on the sinus node, and variability reflects spontaneous changes in autonomic activity [12]. HRV is a simple and important tool for studying the autonomic control of the heart and autonomic dysfunction, and it represents one of the most promising of such markers $[13,14]$. As a development or progression, we suggest the possibility of ANS imbalance and use HRV as a method to evaluate ANS tone.

Previous epidemiological studies in men with $\mathrm{BPH}$ have suggested that a relationship between may exist between sympa- thetic overactivity and LUTS. Meigs et al. [15] noted that men with symptomatic BPH were also more likely to have characteristics known to be associated with increased sympathetic activation, specifically heart disease, $\beta$-blocker use, and a sedentary lifestyle. Hammarsten and Hogstedt [16] also found a relationship between more rapidly developing $\mathrm{BPH}$ and diseases including obesity, increased insulin, increased risk of type 2 diabetes and hypertension.

Choi et al. [17] reported ANS activity in men with voiding symptom-predominant LUTS and storage symptom-predominant LUTS. They showed that patients with LUTS exhibited decreased HF than healthy controls, which indicated that they may had some disease or imbalance in the ANS, that may distinguish LUTS patients from healthy men. However, the LF/HF ratio, which represents ANS balance, was not significantly different between LUTS patients and healthy controls. Thus, in the present study, we divided the LUTS patients into two groups by the median $\mathrm{LF} / \mathrm{HF}$ value of the healthy controls. Because most investigators believe that LF and HF represent sympathetic and parasympathetic nervous system activity, respectively. Previous studies LUTS patients had an increased sympathetic tone, and voiding symptom predominant patients had more increased sympathetic tone. But our results suggest that LUTS patients could have relatively hypoactive sympathetic tone than healthy men, and they suffered from storage symptom more than those with hyperactive sympathetic tone, especially frequency.

Unfortunately, no specific modalities can be used to improve autonomic dysfunction. In some studies, HRV was improved by exercise or cessation of smoking. Accordingly, it may be beneficial for physicians to advise their patients to perform more exercise and to quit smoking $[18,19]$.

This study was limited in that LUTS are not specific and can be changed by factors unrelated to prostatic conditions, including diet, fluid intake, alcohol intake, anticholinergic effects of commonly used over-the-counter medications, smoking and even mood or physical activity [20-22]. We attempted to reduce such effects by asking patients to not consume anything that could affect the ANS before the HRV recording. Second, the sample size of both groups was small: thus, additional study with large numbers will be needed. Last, electrocardiography recording was measured only for 5 minutes in a sitting position and not for 24 hours to simplify the measurement and to reduce the discomfort of the subjects.

Many patients suffer from LUTS that do not respond to current medications or surgical treatments, and we suggest that an 
imbalance in ANS activity may be a factor that cause or progression of diseases in men with LUTS. In particular, LUTS patients with hypoactive sympathetic tone may suffer from frequency and storage symptom by ANS dysfunction. We believe it is time to shift our concern to general health and ANS dysfunction to find the other etiologies of LUTS.

\section{CONFLICT OF INTEREST}

No potential conflict of interest relevant to this article was reported.

\section{REFERENCES}

1. Kupelian V, Wei JT, O'Leary MP, Kusek JW, Litman HJ, Link CL, et al. Prevalence of lower urinary tract symptoms and effect on quality of life in a racially and ethnically diverse random sample: the Boston Area Community Health (BACH) Survey. Arch Intern Med 2006;166:2381-7.

2. McVary KT, Rademaker A, Lloyd GL, Gann P. Autonomic nervous system overactivity in men with lower urinary tract symptoms secondary to benign prostatic hyperplasia. J Urol 2005;174(4 Pt 1): 1327-433.

3. van Ravenswaaij-Arts CM, Kollee LA, Hopman JC, Stoelinga GB, van Geijn HP. Heart rate variability. Ann Intern Med 1993;118:43647.

4. Kim JC, Joo KJ, Kim JT, Choi JB, Cho DS, Won YY. Alteration of autonomic function in female urinary incontinence. Int Neurourol J 2010;14:232-7.

5. Lee JY, Joo KJ, Kim JT, Cho ST, Cho DS, Won YY, et al. Heart rate variability in men with erectile dysfunction. Int Neurourol J 2011; 15:87-91.

6. Cho DS, Choi JB, Kim YS, Joo KJ, Kim SH, Kim JC, et al. Heart rate variability in assessment of autonomic dysfunction in patients with chronic prostatitis/chronic pelvic pain syndrome. Urology 2011;78: 1369-72.

7. Kasturi S, Russell S, McVary KT. Metabolic syndrome and lower urinary tract symptoms secondary to benign prostatic hyperplasia. Curr Urol Rep 2006;7:288-92.

8. Thor PJ, Mazur M, Furgała A, Worek M. The autonomic nervous system function in benign prostatic hyperplasia. Folia Med Cracov 2006;47:79-86.

9. Bushman W. Etiology, epidemiology, and natural history of benign prostatic hyperplasia. Urol Clin North Am 2009;36:403-15.
10. McConnell JD, Roehrborn CG, Bautista OM, Andriole GL Jr, Dixon CM, Kusek JW, et al. The long-term effect of doxazosin, finasteride, and combination therapy on the clinical progression of benign prostatic hyperplasia. N Engl J Med 2003;349:2387-98.

11. Andersson KE. LUTS treatment: future treatment options. Neurourol Urodyn 2007;26(6 Suppl):934-47.

12. Choi JB, Kim YB, Kim BT, Kim YS. Analysis of heart rate variability in female patients with overactive bladder. Urology 2005;65: 1109-12.

13. Task Force of the European Society of Cardiology and the North American Society of Pacing and Electrophysiology. Heart rate variability: standards of measurement, physiological interpretation and clinical use. Circulation 1996;93:1043-65.

14. Silvetti MS, Drago F, Ragonese P. Heart rate variability in healthy children and adolescents is partially related to age and gender. Int J Cardiol 2001;81:169-74.

15. Meigs JB, Mohr B, Barry MJ, Collins MM, McKinlay JB. Risk factors for clinical benign prostatic hyperplasia in a community-based population of healthy aging men. J Clin Epidemiol 2001;54:935-44.

16. Hammarsten J, Hogstedt B. Clinical, anthropometric, metabolic and insulin profile of men with fast annual growth rates of benign prostatic hyperplasia. Blood Press 1999;8:29-36.

17. Choi JB, Lee JG, Kim YS. Characteristics of autonomic nervous system activity in men with lower urinary tract symptoms (LUTS): analysis of heart rate variability in men with LUTS. Urology 2010; 75:138-42.

18. Lai FC, Tu ST, Huang CH, Jeng C. A home-based exercise program improves heart rate variability and functional capacity among postmenopausal women with coronary artery disease. J Cardiovasc Nurs 2011;26:137-44.

19. Harte CB, Meston CM. Effects of smoking cessation on heart rate variability among long-term male smokers. Int J Behav Med 2013 Feb 9 [Epub]. http://dx.doi.org/10.1007/s12529-013-9295-0.

20. Boyle P, Robertson C, Mazzetta C, Keech M, Hobbs FD, Fourcade R, et al. The prevalence of lower urinary tract symptoms in men and women in four centres. The UrEpik study. BJU Int 2003;92:409-14.

21. Fowke JH, Phillips S, Koyama T, Byerly S, Concepcion R, Motley SS, et al. Association between physical activity, lower urinary tract symptoms (LUTS) and prostate volume. BJU Int 2013;111:122-8.

22. Harte CB, Liverant GI, Sloan DM, Kamholz BW, Rosebrock LE, Fava M, et al. Association between smoking and heart rate variability among individuals with depression. Ann Behav Med 2013 Feb 23 [Epub]. http://dx.doi.org/10.1007/s12160-013-9476-8. 